\title{
TECHNIQUES IN ORGANIC MICROSYNTHESIS
}

\author{
V. HorÁk \\ Department of Organic Chemistry, Charles University, Prague, \\ Czechoslovakia
}

\section{INTRODUCTION}

In recent years numerous problems have arisen, in various fields of organic chemistry, in which it is necessary to manipulate microquantities of compounds. This has led to the introduction and development of micropreparative techniques, which have also been successfully adapted to organic chemical problems which could be solved on a classical macropreparative scale. The semi-microtechniques, in general, have the advantage over macrotechniques in simpler manipulation, saving of time and material and greater control over the conditions of the reaction.

This review will cover some of the more recent developments in the art of semi-micro organic preparations and purifications.

\section{GENERAL RULES}

The most important rule for organic microtechniques is to restrict the handling of the undissolved compounds to a minimum; this is especially true when the compound is a liquid as this leads to extensive loss of material. Whenever possible dilute solutions are used. However, the degree of dilution must be carefully considered in relation to the particular phenomenon or procedure.

Some processes are partially or completely independent of concentration e.g., liquid-liquid partition processes, or very sharp separation of acids and bases by ion exchange techniques. However, some chromatographic processes will not give satisfactory results if the sample is placed on an adsorbent column or injected into a gas-chromatographic column dissolved in a large excess of solvent. The zones or peaks will not be sharp and the separation will be less efficient.

It is well-known that for processes involving chemical reactions the concentration is of great importance. No particular concentration effect will be observed if the reaction is of first or pseudo-first order (solvolytic reactions). However, second order reactions have a high concentration dependence because of the lesser probability of two reactive particles meeting and the consequent lowering of the number of active collisions in a given volume as the solution becomes more dilute. In this case there is not only an increase in the reaction time as a result of high dilution, but also a lowering of the yield of the reaction products because of the increasing importance of solvolytic side-reactions ${ }^{1}$ (reactions with solvent or buffer molecules and ions); these are not important under normal conditions, because the main reaction is much faster. It is possible to avoid this problem even in dilute solution by using a large excess of the reagent, and selecting the solvent carefully. Dipolar 


\section{HORAKK}

aprotic solvents ${ }^{2}$ may be especially useful, as in some cases they may increase the reaction rate by a factor of up to $10^{7}$. Alternatively, with for example a capillary technique, concentrated solutions may be used.

\section{LIQUID TEGHNIQUES}

Because the compounds are generally used as dilute solutions it is necessary to become familiar with rapid and quantitative methods for the handling of liquids. Even when dealing with dilute solutions, the volumes may be of the order of only a few hundred microlitres. Pipetting is the preferred technique.

Two types of pipettes are used: those which are open at both ends and those open only at one. The first type requires some kind of suction apparatus. For the second type accessory equipment for filling or dispensing of the liquid is not necessary. Instead, use is made of the expansion and constriction of gas by heating and cooling. One-end sealed pipettes ${ }^{3}$ are specially prepared so that they have the most convenient shape and dimensions. They are advantageous where special handling is necessary and are also very useful for keeping liquids and solutions. If the pipette is sealed on both ends, the sample is protected from attack by atmospheric oxygen, moisture, and carbon dioxide as well as from evaporation of the solvent and volatile compounds.

Pipetting may also be combined with other simple operations. For example, filtration is possible while pipetting if the capillary portion of the pipette is fitted with a cotton plug ${ }^{4}$ or a miniature filter paper disc.

The use of dilute solutions means that the solvent must be evaporated in order to concentrate the solution. The most efficient and most common technique is to evaporate the solvent with a stream of gas. Air ${ }^{5}$ or inert gas (nitrogen and carbon dioxide) are usually used. It is even possible to concentrate a solution in a capillary ${ }^{6}$ by placing a narrower capillary or injection needle inside to serve as a gas inlet. Tank nitrogen is the most convenient source of gas for concentrating solutions without fear of contamination with oxidation products or moisture. The same technique is also used for evaporation of a solution to dryness.

Similar techniques are used for the evaporation of solutions to one spot on porous papers. Sometimes as much as several hundred microlitres can be applied in this fashion. Various techniques and equipment designs have been described to enhance evaporation and still make small spots. Most of these consist of a hot stage with an attachment for placing the paper in a stream of gas? ${ }^{7}$ Spotting on cellulose or glass-fibre paper is a common procedure in chromatography and is also used in those cases where the paper serves as a supporting material, e.g., for reaction procedures, separation of compounds by extraction etc.

\section{REACTION TECHNIQUES}

The main task in any chemical synthetic work is to obtain the highest possible yield. Since in microchemical synthesis only small quantities of reagent are needed it is desirable to use the procedure which consistently gives the highest yield even if the price of the reactant is high and it is not readily available. Microchemical methods are to be most highly recom- 
mended in syntheses with isotopically labelled compounds. Here the choice of the non-labelled portion of the molecule should be such as to guarantee the highest isotopic yield.

In choosing the reagent, the separation (especially chromatographic) and purification of the reaction products should be considered. For separation it is desirable that the product be coloured, fluorescent, isotopically labelled, readily crystallizable, or have characteristic solubility properties.

\section{Semi-microtechniques}

Classical semi-microtechniques are often very similar to those which are familiar in macroscale procedures. The problem of treatment of small samples is solved by the miniaturization of the equipment. There is a large selection of commercially available equipment, which is suitable for the construction of any necessary reaction set-up.

A rather different approach to this problem has been described by Cheronis in his classic book Micro and Semi-microtechniques ${ }^{8}$. The system consists of a cold finger condenser and flask with matching wide ground-glass joints. The reflux condenser extends downwards, so that it is close to the surface of the boiling liquid. The advantage of a wide neck is that material on the walls of the flask can more easily be reached. Another advantage of the system is that the joint is cold because it is not in contact with the vapour from the boiling liquid. Lastly the amount of liquid which is in the vapour phase is smaller than in the system mentioned above.

In some cases improvized equipment is constructed for special purposes. It is often easier to prepare simple equipment from glass tubing, which has many advantages over standard equipment constructed in the usual manner. The building of improvized equipment demands experience and skill on the part of the laboratory worker.

Some points concerning special techniques for the measurement and addition of small amounts of reagents should be made. Some reagents are difficult to add quantitatively in the usual way. For example, in reactions requiring chlorine it is difficult to measure the gas accurately, but it is possible to weigh out a stochiometric amount of potassium permanganate and add an excess of concentrated hydrochloric acid. The gas is prepared in a separate vessel and transferred into the reaction mixture by an inert carrier gas (nitrogen). This technique of slow mixing of volatile reagents transported by a stream of an inert gas has much more general use. It is possible to control the rate of addition of the reagent by the rate of flow of the stream of gas and by the regulation of the temperature of the generating flask. Another precise method is the electrolytic generation of a particular reagent, e.g., small amounts of bromine, thiocyanate, hydrogen and oxygen, using coulometric equipment.

\section{Capillary techniques}

For microscale preparations, where the volume of the reaction mixture is less than $500 \mu$ l, the sealed capillary technique is to be highly recommended. The reaction mixture is transferred into a glass tube of a suitable length and inner diameter which is then sealed at both ends. The reaction is usually 


\section{HORAKK}

carried out in a hot water bath. In this way it is possible to treat volumes from a few microlitres up to several hundreds of microlitres. This technique has the advantage, from the point of view of microscale preparations, of being very simple, requiring no special equipment and protecting the reaction mixture from moisture, oxygen etc. Usually there are no special requirements for the quality of glass or thickness of the walls of the tubing used. Capillaries of larger diameter may be filled after sealing at one end. Very narrow capillaries are more easily filled whilst still open at both ends. Small capillaries may also be sealed at one end, after which the open end is immersed into the reaction mixture. The mixing tube and the capillary are placed in a vacuum chamber and the chamber evacuated. The capillary is filled by releasing the vacuum. After the reaction is completed it is advisable to cool the capillary before opening. If necessary the liquid can be transferred from one end of the sealed capillary to the other by shaking or centrifugation.

Other microscale operations can be performed using capillary techniques. Liquid-liquid extraction using the reaction capillary is advisable only if the inside diameter is greater than $2 \mathrm{~mm}$. The extraction liquid is injected into the capillary with a syringe. The capillary is then sealed and vigorously shaken. Separation of the layers may be improved by centrifugation. The extraction liquid is taken up with a syringe fitted with a long thin needle. It is possible to repeat the process several times. Extraction in capillaries of smaller diameter is a much more difficult procedure. In such a case other micro liquid-liquid extraction techniques must be used even if a higher dilution of the solution is necessary.

Separation of crystals and repeated recrystallization in capillaries has also proved to be a very useful technique. Crystals are collected on the bottom of the tube by centrifugation. The supernatant liquid is collected with a syringe, after which the capillary is refilled with a suitable solvent, resealed and heated. It is preferable to use a suitable solvent rather than to try to cool the solution with dry ice. Many low boiling solvents which are used in sealed capillaries for crystallization (ether, tetrahydrofuran) show excellent solvent properties at higher temperatures above their boiling point. Finding a suitable solvent is much simpler when using capillaries, as solvent which does not have the correct properties may easily be evaporated off.

Preparative gas chromatography has taken the place of the Morton capillary microdistillation technique ${ }^{9}$.

\section{Reaction techniques combined with chromatography}

Cassidy ${ }^{10}$ writes of the chemical changes which have been observed during chromatographic processes. These are especially true of adsorption chromatography on alumina and charcoal, and include hydrolysis, isomerization, oxidation etc. These are limitations for which precautions should be taken.

However, the same phenomena can also be of value in preparative work, and the chromatographic procedure arranged to give the most quantitative yield of the particular desired product. This method is well suited to small quantities of compounds.

Techniques, which are readily combined with chromatographic procedures, but are not in themselves chromatographic, will also be included in this section. 
Paper techniques

Microchemical reactions may be performed on paper in a variety of ways. The techniques were originally developed from studies of paper chromatography. These reactions may or may not be used solely for the detection of a compound. Reaction may be completed before the actual chromatography, preceding the liquid phase development.

A very interesting combination of paper chromatography with a specific reaction procedure has been described as follows ${ }^{11 a}$. The sample (a mixture of several compounds) is spotted at the bottom of a sheet of chromatographic paper for use in two-dimensional chromatography. After developing and drying the paper, the reagent is applied. The development in the second direction with the same liquid system brings compounds which have not reacted with the reagent to the diagonal. Spots not falling on this line are then reaction products.

These techniques are not only applicable to analyses but also make possible the extraction of spots for the isolation of pure reaction products.

Both cellulose and glass-fibre papers have been used as a base for these reactions. The glass-fibre papers are especially useful, because they may be used with very reactive reagents, e.g., strong mineral acids and hydroxides, elementary halogens and ozone.

Volatile reagents may be preferred because the size of the spot is not changed during the process and the excess of the reagent is easily removed by evaporation in air, after the paper has been kept the necessary time in a saturated atmosphere of the reagent in a suitable container. Catalytic hydrogenation has been accomplished in this way by preparing the catalyst on the bottom of the paper before applying the compound and hydrogen gas $^{11 b}$. Other reagents which can be applied in the vapour phase are $\mathrm{HCl}$, $\mathrm{HNO}_{3}, \mathrm{HCOOH}, \mathrm{SO}_{3}, \mathrm{SO}_{2}, \mathrm{~N}_{2} \mathrm{O}_{4}, \mathrm{CO}_{2}, \mathrm{HCOOOH}, \mathrm{O}_{3}$, elementary halogens and thiocyanate, $\mathrm{SO}_{2} \mathrm{Cl}_{2}, \mathrm{CH}_{2} \mathrm{~N}_{2}, \mathrm{H}_{2} \mathrm{~S}$, and $\mathrm{CH}_{3} \mathrm{I}$. In some cases the reaction proceeds very slowly, when it is possible to place the paper and reagent in a small autoclave so that the compound can react at a higher temperature ${ }^{12}$.

The spraying of liquid reagents has also been successfully used in chemical reactions with compounds spotted on a paper. Among the reagents which have been used are $\mathrm{HIO}_{4}, \mathrm{~Pb}(\mathrm{AcO})_{4}, \mathrm{CrO}_{3}, \mathrm{CrO}_{3}+\mathrm{Ac}_{2} \mathrm{O}, \mathrm{LiAlH}_{4}$, $\mathrm{NaBH}_{4}$, triphenyl tetrazolium chloride and Oppenauer reaction reagent ${ }^{13}$.

\section{Adsorption and ion exchanger techniques}

The hydrolysis of esters is a reaction which occurs readily especially on a column of alkaline alumina ${ }^{10}$. It proceeds under normal conditions on passing of the ester solution through the column. This technique is used for the liberation of the alcohol from its ester derivative but is not satisfactory for the liberation of the acid moiety; this is bound firmly to the alumina by ionic bonds. The procedure is simplified if the particular acid is coloured, since the whole procedure can then be easily followed visually.

Ion exchangers have been similarly applied in chromatographic procedures, e.g., esters of sulphonium alcohols come out as the corresponding alcohols when passed through strongly basic ion exchanger columns ${ }^{14}$. Water must be used as the solvent for this procedure (in alcohol no 


\section{HORÁK}

hydrolysis is observed), while when alumina is used as the stationary phase, solvents which are even less polar may be used.

While ester hydrolysis on chromatographic columns seems to be often used, other reactions which are observed during chromatography ${ }^{10}$ do not appear to be generally useful. More systematic studies of this method would be very helpful.

Quantitative yields are observed if molecular complexes, as of the type aromatic hydrocarbon-picric acid (charge transfer complexes) are decomposed on chromatographic columns of alkaline alumina ${ }^{15}$. The picric acid, as well as other $\pi$-acids, is firmly bound, while hydrocarbons readily pass through the column.

Ion exchangers can be highly recommended as catalysts ${ }^{16}$ for chemical reactions on a micro scale. They have the advantages of mild action and easy handling, especially in separating the resin from the reaction mixture.

\section{Gas chromatography}

Gas chromatographs have been successfully combined with the reaction equipment. Chemical compounds, or their mixtures, are changed by chemical processes and the reaction products are directly transferred on the column. Three types of equipment are used. One uses dissolved reactants in a liquid phase, the second proceeds in the gas phase, and the last proceeds under pyrolytic conditions. Examples of these are $(i)$ the desulfurization of organic sulfur compounds by Raney nickel in n-butanol solution ${ }^{17}$, (ii) gas phase catalytic hydrogenolysis in which hydrogen is used as the carrier gas ${ }^{18}$, and (iii) pyrolytic decomposition of polymers on a hot wire ${ }^{19}$.

\section{SEPARATION AND PURIFICATION OF REAGTION PRODUGTS}

If the micropreparative step of synthetic reactions is not directly combined with a separation procedure, it is necessary to use a suitable technique for the isolation of the pure reaction products.

\section{Crystallization}

Crystallization is a classical laboratory technique whose importance on the microscale has not been diminished by the development of newer methods. The capillary crystallization technique, mentioned above, is recommended for the purification of milligram quantities of material. The technique is more generally useful than that of crystallization on a microscope slide ${ }^{8}$.

The advantage of the automatic hot filtration technique for crystallization on a micro scale may be mentioned here ${ }^{20}$. This procedure has developed from the one-end sealed pipette technique. Before crystallization, the hot solution is filtered through the cotton plug by a simple one- or two-piece automatic suction apparatus. The suction is caused by the cooling of the solvent vapours after all the air has been expelled by boiling. What is usually the most tedious step of crystallization takes only a few seconds.

\section{Distillation}

Distillation is still a difficult method in microscale experimentation.

For semi-microquantities (hundreds of microlitres) the Hickmann distillation flask technique still seems to be the simplest and the most efficient ${ }^{3}$. 


\section{TECHNIQUES IN ORGANIC MICROSYNTHESIS}

There are various modifications of the simple type of Hickmann flask. The advantages of this technique are the short vapour path, easy handling, and the most quantitative yield. Pipetting is recommended for the introduction of samples, as well as for the removal of distillation fractions.

Capillary columns with properly prepared inner surfaces are necessary for fractional microdistillation. The polished surface of the glass capillary ( $2 \mathrm{~mm}$ internal diameter) is mechanically or chemically (hydrogen fluoride) etched ${ }^{21}$. The refluxing material forms a better liquid film on the etched rather than the smooth glass. The efficiency of the column is high. Under optimal conditions it will give about 33 theoretical plates for a $1 \mathrm{~m}$ column.

Although some progress has been made in column microdistillation, this method, in general, does not compare with preparative gas chromatography. The Morton capillary microdistillation technique has been previously discussed from this point of view.

\section{Sublimation}

Sublimation has, in very extensive applications, been shown to be one of the most convenient and quantitative methods for the purification of organic compounds on a micro scale. The apparatus usually consists of a cold-finger condenser in a deep vessel or a hot-stage microscope and a vacuum system.

The introduction of temperature gradient cooling apparatus meant a great improvement in sublimation techniques. Sublimation became not only a powerful method of purification, but also of separation. There are two types of equipment in use. In one of them the sample is sublimed in a narrow tube in a high vacuum ${ }^{22}$, while in the second, vapours of the compounds are flushed through the tube by an inert carrier gas ${ }^{23}$.

\section{Liquid-liquid partition and extraction}

As the liquid-liquid partition process is not limited by concentration and can sometimes be improved by the salting out of the compound to be extracted, there are no particular demands on the size of the equipment. Apparatus with convenient properties, such as a polythene separating funnel, simplifies the process ${ }^{24}$. The layers automatically separate with the water going to the rounded portion and the organic phase to the capillary.

The extraction of spots from cellulose or glass-fibre papers may be a good substitute for liquid-liquid partition. The technique for the quantitative extraction of spots, which is familiar from paper chromatography, can be used. As it is readily possible to change solvents, no difficulty is encountered in performing a gradient elution with solvents of increasing polarity 25 .

\section{Chromatography}

Chromatography has so many applications as a convenient micromethod for preparative separation of organic compounds, that it is impossible to give a comprehensive review here. One technique only has been selected for consideration.

On a micro scale, thin-layer chromatography with a free adsorbent can successfully be substituted for adsorption column chromatography ${ }^{26}$. The dry powder is evenly spread on a glass plate with a glass rod equipped with 


\section{HORÁK}

two rubber bungs. Because the cohesion of adsorbent particles is small, careful treatment of the plate is necessary, but on the other hand the sample spot is easily isolated. The most convenient method is the direct transfer of adsorbent particles from a particular part of the chromatogram into the extraction tube by suction. This technique has the advantage that the precise separation and collection of spots after the development of the whole chromatogram, or of only one side of it, is substituted for the inconvenient collection of small fractions. The capacity of the layer can be varied slightly by varying its thickness.

The selection of the above methods has been partially influenced by the experience of several laboratories in Czechoslovakia, where extensive use of organic microchemical techniques has been made and where some have been developed. Two institutions should be mentioned in this respect: The Institute of Organic Chemistry and Biochemistry of the Czechoslovakian Academy of Sciences in Prague, where these methods have a long tradition, and the Department of Organic Chemistry of Charles University in Prague, where they have been introduced into the curriculum ${ }^{27}$.

\section{References}

1 A. A. Frost and R. G. Pearson. Kinetics and Mechanism, p. 160, Wiley, New York (1963).

2 A. J. Parker. Quart. Rev. (London) 16, 163 (1962).

3 V. Herout. Laboratoriumstechnik der organischen Chemie (translation from Czech) (Ed. B. Keil), chapter XV, Akademie Verlag, Berlin (1961).

4 Pan Huo-Ping. Mikrochim. Acta 1958, 713.

5 G. W. Perold. Mikrochim. Acta 1959, 251.

6 E. Schneider and F. Schneider. Microchemical Laboratory, Wiley, New York (1932).

7 G. Gorbach and G. Gallob-Hausmann. Mikrochim. Acta 1962, 102.

8 N. D. Cheronis. "Micro and Semi-micro Methods." In Techniques of Organic Chemistry (Ed. A. Weisberger), Vol. 6, Interscience, New York (1954).

9 A. A. Morton and J. F. Mahoney. Ind. Eng. Chem. (Anal.) 13, 494 (1941).

10 H. G. Cassidy. "Adsorption and Chromatography." In Techniques of Organic Chemistry (Ed. A. Weisberger), Vol. 5, Interscience, New York (1951).

11a O. Mikeš and V. Holeyšovský. Chem. Listy 51, 1497 (1957).

11b H. P. Kaufman and D. K. Chowdhury. Chem. Ber. 91, 2117 (1958).

12 V. Prey, A. Kabil and H. Berbalg. Mikrochim. Acta 1959, 68.

13 L. R. Axelrod. Anal. Chem. 27, 1308 (1955).

P. J. Peterson and G. W. Butler. J. Chromatog. 8, 71 (1962).

H. P. Kaufman and H. J. Hennick. Mikrochim. Acta 1961, 333.

14 V. Horák. Unpublished results.

15 P. A. Plattner and A. St. Pfau. Helv. Chim. Acta 20, 224 (1937).

16 R. Griessbach and G. Neumann. Chem. Tech. (Berlin) 5, 187 (1953).

17 J. Franc, J. Dvořáček and V. Koloušková. Mikrochim. Acta 1965, 4.

18 C. J. Thompson, H. J. Coleman, R. L. Hopkins, and H. T. Rall. Anal. Chem. 37, 1042 (1965).

${ }^{19}$ J. Janák. Collection Czech. Chem. Commun. 25, 1780 (1960).

J. R. Dhout. Nature 192, 747 (1961).

20 M. Svoboda. Chem. Listy 53, 31 (1959).

V. Horák. Chem. Listy 54, 723 (1960).

21 A. J. Levin. Zh. Prikl. Khim. 31, 1655 (1958).

22 T. H. Bates. Chem. Ind. (London) 1958, 1319.

G. Schmidt. Mikrochim. Acta 1959, 406.

${ }_{23}$ W. H. Melhuish. Nature 184, 1933 (1959).

24 H. Marchart. Mikrochim. Acta 1962, 913.

25 V. Prey and A. Kabil. Mikrochim. Acta 1959, 79.

26 Ž. Procházka. Chem. Listy 55, 974 (1961).

27 V. Horák. Prace s malymi kvanty v organicke laboratori (The Work with Small Quantities in the Organic Laboratory; published in Czech), SNTL, Prague (1964). 